Volume 6, Issue 1, 1-6 Pages

Research Article | Open Access

ISSN (Online)- 2380-5706

DOI : $10.21694 / 2380-5706.21002$

\title{
Evaluation of Phytochemical Constituents and Sedative Activity of Passiflora Edulis Sims Extracts
}

\author{
Hung Tran - Quoc ${ }^{1}$, Trinh Tang-Le-Quynh ${ }^{1}$, Nguyet Do-Thi, Chi Nguyen-Thi-Lan, Toi Nguyen-Van, Ha \\ Le-Thi-Ngoc, Tram Tang-Thi-Thao, Anh Nguyen-Tuan, Ha Ngo-Duy-Tuy, Sy Duong-Quy ${ }^{1,2 *}$ \\ ${ }^{1}$ Medical - Biological Research Center, Lam Dong Medical College, DaLat City, Vietnam. \\ ${ }^{2}$ Penn State Medical College, Hershey Medical Center, PA, USA.
}

\begin{abstract}
Passiflora edulis Sims, Passifloraceae is widely grown in Lam Dong, Vietnam for its fruit as a beverage. This study is an attempt to explore the phytochemical constituents using the chemical reagents and evaluate the sedative, anti - anxiety activity of ethanol and water extract of leaves and rhizomes using the elevated plus-maze model and dark-light box test in mice. The phytochemical screening results showed the presence of alkaloids, tannins, phenolic compounds, flavonoids in leaves and rhizomes of Passiflora edulis, and the leaf have more alkaloids and flavonoids than the rhizomes. Moreover, treatment with the leaf extract significantly $(p<0.001)$ demonstrated sedative, anti-anxiety activity as compared to vehicle control, in which the activity of ethanol extracts was significantly $(p<0.001)$ better than water extracts. In addition, at dosage of $300 \mathrm{mg} / \mathrm{kg}$ the leaf ethanol extracts showed significantly $(p<0.001)$ lower than diazepam $2 \mathrm{mg} / \mathrm{kg}$. These finding are an important factor in evaluating the pharmacological effects and orienting the process of preparing sedative tea products using Passiflora edulis leaf grown in Lam Dong, Vietnam in the future.
\end{abstract}

KEYWORDS: anti-anxiety, phytochemical, elevated plus-maze, dark - light box, sedative Passiflora edulis Sims

\section{INTRODUCTION}

Anxiety disorder and insomnia, the fairly common disease characterized by cognitive, emotional, and behavioral components, affect one-eighth population worldwide and have become an important research area in the field of psychopharmacology [1]. Nowadays, besides the use of psychotherapy, the use of sedatives such as benzodiazepines (sedusen,zolpidem...), barbituratederivatives (phenobarbital ...) come with sanative potential to manage sleeping disorders, and may also reduce anxiety [2]

However, some side effects associated with these drugs, such as insomnia, muscle relaxation and hepatotoxicity were reported [3] have made it limited for use in patients. Therefore, many researchers are trying to find a newer, more effective, and more tolerable alternative medicine. During the research and development of anxiety therapies, several traditional medicinal plants have been discovered to be effective against animal anxiety. [4]

Passiflora edulis Sims (PE), a perennial vine, has many good nutritional properties, is used as raw materials for processing special flavored beverages, foods, and medicines that are very beneficial to human health. The primary phytochemicals found in the passionflower are flavonoids such as vitexin, chrysin, luteolin, apigenin, kaempferol and quercetin derivative; alkaloids such as harman, harmine, harmaline, harmol and harmalol; polyphenols, triterpenes, and its glycosides. Some other isolated plant constituents have been identified such as glycosides, carbohydrates, amino acids, benzopyrones, carotenoids, cyanogenic glycosides, polysaccharides. [5-7]

In traditional medicine, The PE has been used extensively for the treatment of some diseases like diuretic, anthelmintic, antidiarrheal, stimulant, tonic, and also in the treatment of hypertension, menopausal symptoms. On the other hand, PE showed positive effects in episodes of anxiety, insomnia, sleeplessness, and in depressive states. The use of PE in patients with chronic insomnia can be helpful in controlling sleep disorders, dementia, and degenerative brain diseases caused by insomnia through a sedative effect, thereby helping to improve a patient's chronic insomnia. The literature data suggest that the passionflower itself, as well as preparations, helps reduce stress and be helpful in the treatment of insomnia, anxiety, and depression. [5]

Consequently, it seems to be a potential, effective and safe medicine in the treatment of stress reactivity, insomnia, 
anxiety, and depression-like behaviors. This influenced us to design and conduct the present study to define the phytochemical and anxiolytic effect of this plant. In the present study, we have determined the phytochemical of PE extract and evaluated the efficacy of PE preparations in the treatment of insomnia and anxiety using Elevated Plus-Maze - EMP and Light/dark model.

\section{MATERIALS AND METHODS}

\section{Plant Collection and Preparation}

Passiflora edulis Sims, Passifloraceae were collected in Tram Hanh, Dalat, Vietnam and identified by Medical - Biological Research Center, Lam Dong Medical College, then divided into 2 parts: aerial parts (A) and rhizomes (R) and washed to remove any dirt. It was cut into small pieces, dried in the sunlight for seven days and kept in an oven at $40^{\circ} \mathrm{C}$ for 12 hours, and ground into a coarse powder, which was stored in an airtight container and kept in a cool, dark, and dry place at room temperature in the Faculty of Pharmacy of Lam Dong Medical College until further preparations.

\section{Extraction of Plant Materials}

$100 \mathrm{~g}$ of each coarse powder plant part was macerated in 500 $\mathrm{mL}$ of different solvents (ether ethylic, ethanol $90^{\circ}$, distilled water) in a 2 litter round bottom flask and kept for 48 hours before filtering, the plant solution was then filtered. The resulting filtrate was evaporated and concentrated to a paste dryness using water bath set at $45^{\circ} \mathrm{C}$ to obtain the extract then was stored in the refrigerator below $10^{\circ} \mathrm{C}$ until required for further analysis.

\section{Qualitative Phytochemical Analysis of Leaf and Rhizomes Parts of PE in Different Solvents}

The ether ethylic, ethanol $90^{\circ}$, distilled water of leaf and rhizomes prepared extracts were analyzed for phytochemical constituents (phenol, alkaloids, flavonoids, etc.) using standard qualitative methods described by Tiwari et al. [8] with some modifications for detecting the presence of secondary metabolites: alkaloids, phenol, flavonoids, carbohydrates, triterpenes, saponin, and tannins. The visible color change or precipitate formation was taken into consideration for $(++)$ strongly present, $(++)$ present or $(-)$ absent of particular active constituents. The values obtained are triplicates for all secondary metabolites determined.

\section{Anxiolytic Evaluation}

\section{Animals}

Swiss mice of either sex, weighing 20 - 24g obtained from Pasteur Institute of Nha Trang, were employed in the present study in different groups, each consisting ten mice. One group was used as control (saline, p.o), second for standard drug (diazepam $2 \mathrm{mg} / \mathrm{kg}$, p.o) treatment, third, fourth, and fifth group for PE ethanol extract treatment (Test - 100, 200, $100 \mathrm{mg} / \mathrm{kg}$, p.o), sixth, seventh, eighth for PE aqueous extract treatment (Test - 100, 200, $100 \mathrm{mg} / \mathrm{kg}$, p.o). The animals were kept in standard cages and were maintained at room temperature with a natural day and night cycle. They were stabilized and allowed free access to food and water during the study period under the laboratory standard for at least one week prior to testing. Experiments were conducted from 9:00 to 16:00 in Medical - Biological Research Center, Lam Dong Medical College

\section{Elevated Plus-Maze - EMP}

The Elevated Plus maze was built according to the description of Lister (1987) [9] . The model comprises of a plus sign maze having central platform with four arms: two closed (30 $\times 5 \times 15 \mathrm{~cm}$ high walls $)$ and two open arms $(30 \times 5 \mathrm{~cm} \mathrm{x} 4$ $\mathrm{mm}$ high walls to prevent the mice from slipping and falling off the edge) with a central arena $(5 \times 5 \mathrm{~cm})$. The maze was elevated to a height of $50 \mathrm{~cm}$ above the floor. Tests were performed in an isolated quite room to avoid any type of turbulence in the behavior of animals and lit by a 60 - watt red lamp for background lighting. During the experiment each mouse was placed in the central compartment facing one of the open arms. The number of times the animal entered into both, open and closed arms and duration(s) of the same were documented for $5 \mathrm{~min}$. An entry was counted when all four paws of the mouse entered an open or closed arm. An increase in open arms entries and increase in time spent in open arms were interpreted as an index of potential anxiolytic activity.

\section{Light-Dark Model - LDM}

LDM comprises of a rectangular box $(25 \mathrm{~cm} \times 25 \mathrm{~cm} \times 25 \mathrm{~cm})$ consists of two parts, dark compartment was covered from top and was pasted with black chart from inside, while lit compartment was open, pasted with white chart from inside, and was illuminated with $40 \mathrm{~W}$ light source kept $25 \mathrm{~cm}$ above the open box. The mice were allowed to move from one compartment to the other through a transit hole (5 $\mathrm{cm} \times 5 \mathrm{~cm}$ ) in the bottom of the clapboard between the two compartments. An entry was counted when all four paws of the mouse entered an open or closed arm. An increase in open arms entries and increase in time spent in open arms were interpreted as an index of potential anxiolytic activity. The mice were treated with drugs and vehicles as respective groups and after one hour of treatment the mice were put into the center of the light compartment with their back to dark compartment and then transition behavior over 5 min was observed. Number of crossings between the light and dark area and total time spent in the illuminated part of the box were calculated. Every time before placing each animal, the maze was cleaned with $5 \%$ alcohol to eliminate the possible bias due the odor left by the previous animal [10].

\section{Statistical Analysis}

All the results were expressed as mean \pm SEM $(n=10)$. The data were analyzed statistically using one-way analysis of variance (ANOVA) followed by the Tukey's post hoc test for multiple comparisons. $\mathrm{P}<0.05$ was taken to be statistically 
significant. Statistical analysis and graphing were done using GraphPad Prism 8 for Windows version 8.0.2.

\section{RESULT}

\section{Phytochemical Screening}

The phytochemical screening of three different solvent extracts of each parts recorded (Table 1) presence of chemical constituents and showed that ethanol and water extract revealed the presence of alkaloids, phenolic, flavonoids, glycosides and carbohydrate while ether ethylic extract just showed the presence of alkaloids. The results also recorded maximum phytochemical constituents in ethanol extract than aqueous and ether ethylic extract. In addition, the leaf have a strong presence of alkaloids and flavonoids than rhizomes.

According to some references, flavonoids are reported to be the main constituents of PE such as apigenin, luteolin, kaempferol, quercetin and flavonoid glycosides such as vitexin, chrysin $[5,7]$. Among those chemical constituents, Vitexin is a known flavonoids that have been shown protective effects on different models of neurological and psychiatric disorders, including ischemia injury and learning deficit. [11]. Chrysin has been hypothesized to decrease anxiety via interaction with the GABA (A) receptor in laboratory rats as measured by elevated plus-maze assays [12]. Apigenin seems likely helping the shortening of sleep latency through binding to benzodiazepine receptors in the brain [13]. On the other hand, flavonoids such as kaempferol and quercetin in polar herbals extract have reported that glycosides derived of quercetin and kaempferol are possibly potentially responsible for the sedative and anxiolytic-like response [14] [15]. The indole alkaloids based on the beta-carboline ring system such as harman, harmin, harmalin, harmol and harmalol were found in PE and harmane was showed antianxiety and antidepressant effects in rats its mechanism may involve dopamine and serotonin [16]. These findings strongly suggest that PE is a herbal product having anxiety and insomnia in animals and help us to focus on samples of leaf ethanol and aqueous extracts, which have the most chemical constituents, especially flavonoids and alkaloids, for further analysis in the pharmacological evaluation research for the next stage.

\section{Elevated Plus-Maze - EMP}

EPM is one of the most widely test currently used to evaluate the sedative and anxiolytic effects of drugs [17], [18]. This experiment is based on the mouse's instinct to be defensive and to love to explore. In the EPM test, the self-defense instinct manifests itself by seeking shelter in the closed arm, while the discovery instinct manifests itself by entering the open arm. Normally, when placed in an Elevated Plus-Maze instrument, due to height anxiety, rats have less enter open arm areas and often seek safe zone in closed arms. However, when taking medication to reduce anxiety, rats no longer have a fear of heights and will prefer to go out with open hands, due to their instinct to love to explore.
ANOVA of the number of entries and the times spent by mice in the open arms (Table 2 and Figure 3.) of elevated plus maze test showing significant $(\mathrm{P}<0.01)$ differences between the groups. Post hoc comparisons made with Tukey's test revealed animals that received ethanol leaf extract of PE (200$300 \mathrm{mg} / \mathrm{kg}, \mathrm{p.o}$ ) and aqueous leaf extract of PE (200-300 $\mathrm{mg} / \mathrm{kg}$, po.), and diazepam $2 \mathrm{mg} / \mathrm{kg}$ increased significantly $(\mathrm{P}<0.001)$ the number of entries and the time spent in the open arms compared to the control group but interestingly not at $100 \mathrm{mg} / \mathrm{kg}$ of all ethanol and aqueous extracts.

The number of entries and the time spent in the open arm of the group treated with ethanol leaf extract of PE at 300 $\mathrm{mg} / \mathrm{kg}$ body weight was higher $(\mathrm{p}<0.001)$ than that of the treatment with lower doses $(100,200 \mathrm{mg} / \mathrm{kg})$ and aqueous leaf extract of PE at $100-300 \mathrm{mg} / \mathrm{kg}$ body weight but lower $(\mathrm{p}<0.001)$ than Diazepam at $2 \mathrm{mg} / \mathrm{kg}$ body weight. Furthermore the number of entries of the group treated with ethanol extract of PE at $200 \mathrm{mg} / \mathrm{kg}$ body weight did not show the different significantly ( $p>0.1$ ) than aqueous leaf extract of PE at $200 \mathrm{mg} / \mathrm{kg}$ body weight and aqueous extract of PE at $300 \mathrm{mg} / \mathrm{kg}$ body weight treatment.

In our EPM study, the Ethanol and aqueous leaf extract at $200-300 \mathrm{mg} / \mathrm{kg}$ showed significantly an anxiolytic effect by increasing the time spent on open arms and the number of entries in open arms, compared to the control group. The effect of ethanol leaf extract $300 \mathrm{mg} / \mathrm{kg}$ PE on the EPM test was highest but lower than $2 \mathrm{mg} / \mathrm{kg}$ diazepam. In addition, in doses of 200, and $300 \mathrm{mg} / \mathrm{kg}$ for identifying antianxiety, PE leaf ethanol extract significantly $(p<0.01)$ increases the time spent and number of entries in the open arm as compared to control. As the dose of PE increases, the effect also increased at doses of 200 , and $300 \mathrm{mg} / \mathrm{kg}$ of ethanol leaf extract.

\section{Light-Dark Model - LDM}

In addition, the effect of PE was also evaluated using LDM, a popular screening tool in research of anxiolytic or anxiogenic agents [25]. According to Bourin and Hascoet [10], the light/ dark test is based on the innate aversion of rodents to brightly illuminated areas and on the spontaneous exploratory behavior of rodents in response to mild stressors including novel environment and light. Accordingly, this test may be useful to predict anxiolytic-like or anxiogenic-like activity in mice.

The ANOVA of the time spent (s) in lighted box in LightDark Model (Table 3 and Figure 2.) test showing significant $(\mathrm{P}<0.001)$ differences between the groups but there were no significantly differences in the number of transition was observed. In the light/dark exploration test, the time spent (s) in lighted box increased significantly $(\mathrm{P}<0.001)$ in groups treated by ethanol and aqueous of PE extract at dose 200$300 \mathrm{mg} / \mathrm{k}$, and diazepam $2 \mathrm{mg} / \mathrm{kg}$ compared to the control group.

The ethanol and aqueous extract at doses of $200-300 \mathrm{mg} / \mathrm{kg}$ significantly $(\mathrm{p}<0.01)$ increased the time spent in the light 
box with peak effect at the doses of $300 \mathrm{mg} / \mathrm{kg}$ of ethanol extract compared to control (25.70 \pm 3.40 seconds) but lower than Diazepam (2mg/kg p.o). There was no significantly difference ( $p>0.1$ ) between the three groups, ethanol leaf extract $200 \mathrm{mg} / \mathrm{kg}$ and aqueous leaf extract 200 and 300 $\mathrm{mg} / \mathrm{kg}$.

The present study demonstrated that the oral administration of PE at 200 and $300 \mathrm{mg} / \mathrm{kg}$ doses could significantly increase the time the animals spent in the lighted area without altering the total number of transitions in between the compartments. As expected, diazepam also exhibited similar patterns of effects of PE in these models. Due to the genetic or strain variation of the animals used in LDM studies, it has been concluded that simply the time spent in the lighted area, but not the total number of transitions [19]. Therefore, our results suggest that PE may possess anxiolytic potentials along with its sedative properties. Although this research has reached its goals, it should be tested at higher doses than $300 \mathrm{mg} / \mathrm{kg}$ in future studies.

Besides, we should be cautious in extrapolating the dose obtained from animal studies to humans, it may be predicted that the effective dose for a $60 \mathrm{~kg}$ adult man would be 1.5 $g$ dry ethanol extract of PE base on the body surface area (BSA) method [20]. This corresponds to an infusion of approximately $29 \mathrm{~g}$ of PE leave in $100 \mathrm{ml}$ ethanol, considering the yield of the extract. However, the optimum therapeutic dose for human would require further studies, evaluating the effect of the extract in a clinical situation.

Table 1. Results of phytochemical screening for extracts of different plant parts of Passifloraedulis

\begin{tabular}{|c|c|c|c|c|c|c|c|c|c|c|c|}
\hline Parts plan & Extract & Yields (\%) & Alk & Fla & Phe & Anth & Sapo & Gly & Tan & Car & Cou \\
\hline \multirow{3}{*}{ Leaf } & Aqueous & 4.32 & + & + & ++ & - & - & + & - & + & - \\
\hline & Ethanol & 5.23 & ++ & ++ & ++ & - & - & + & - & + & - \\
\hline & Ether ethylic & 2.26 & + & - & - & - & - & - & - & - & - \\
\hline \multirow{3}{*}{ Rhizome } & Aqueous & 3.78 & + & + & + & - & - & + & - & + & - \\
\hline & Ethanol & 4.85 & + & + & + & - & - & + & - & + & - \\
\hline & Ether ethylic & 2.43 & + & - & - & - & - & - & - & - & - \\
\hline
\end{tabular}

Alk: Alkaloids, Fla: Flavonoids, Phe: Phenolics, Anth: Anthranoids, Sapo: Saponins, Car: Carbonhydrat, Tan: Tannins, Cou: Coumarins, - negative test, + positive test. $(++)$ strongly present, $(++)$ present or $(-)$ absent

Table 2. The time spent and number of entries by mice on EPM in open and closed arms

\begin{tabular}{|l|l|l|l|}
\hline & Treatment & Time spent on open arms(s) & Number of entries on open arms (s) \\
\hline 1. & Control (Saline p.o) & $6.900 \pm 0.77$ & $1.80 \pm 0.25$ \\
\hline 2. & Diazepam $(2 \mathrm{mg} / \mathrm{kg} \mathrm{p.o})$ & $78.00 \pm 2.66$ & $9.30 \pm 0.70$ \\
\hline 3. & Ethanol $300(300 \mathrm{mg} / \mathrm{kg} \mathrm{p.o})$ & $65.60 \pm 1.65$ & $6.80 \pm 0.42$ \\
\hline 4. & Ethanol $200(200 \mathrm{mg} / \mathrm{kg} \mathrm{p.o})$ & $34.70 \pm 1.15$ & $4.30 \pm 0.37$ \\
\hline 5. & Ethanol $100(100 \mathrm{mg} / \mathrm{kg}$ p.o $)$ & $7.70 \pm 0.817$ & $1.90 \pm 0.31$ \\
\hline 6. & Aqueous $300(300 \mathrm{mg} / \mathrm{kg}$ p.o $)$ & $40.30 \pm 1.19$ & $4.50 \pm 0.50$ \\
\hline 7. & Aqueous $200(200 \mathrm{mg} / \mathrm{kg}$ p.o $)$ & $28.50 \pm 2.09$ & $3.90 \pm 0.38$ \\
\hline 8. & Aqueous $100(100 \mathrm{mg} / \mathrm{kg}$ p.o $)$ & $7.100 \pm 1.70$ & $2.80 \pm 0.55$ \\
\hline Results are expressed as mean \pm S.E.M., $\mathrm{n}=10$ each group; $* * * \mathrm{p}<0.001$ vs. control; $* * \mathrm{p}<0.05$ vs. control \\
\hline
\end{tabular}
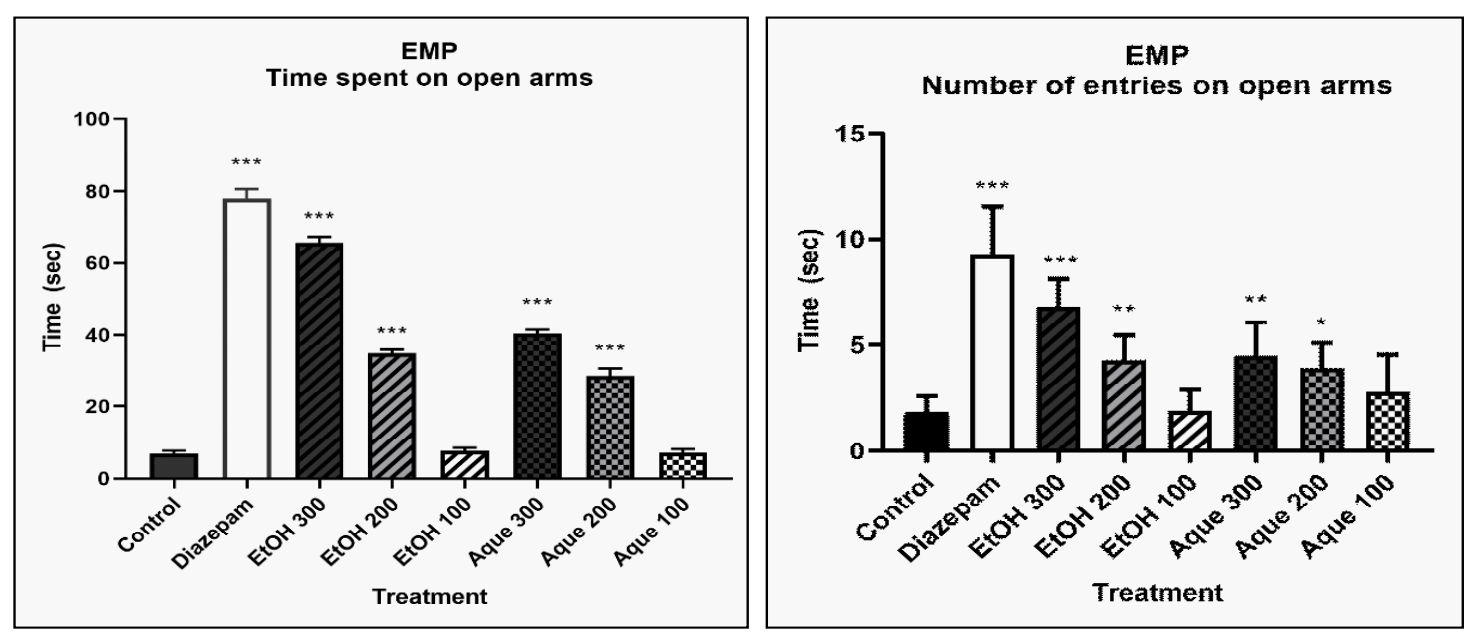

Figure 1. Elevated Plus-Maze test 
Table 3. Light dark test

\begin{tabular}{|c|c|c|c|}
\hline & Treatment & Time in the light box & No. of transition \\
\hline 1 & Control (p.o) & $25.70 \pm 3.40$ & $15.50 \pm 1.26$ \\
\hline 2 & Diazepam (2mg/kg p.o) & $123.3 \pm 6.26$ & $17.70 \pm 2.14$ \\
\hline 3 & Ethanol 300 (300 mg/kg p.o) & $96.20 \pm 3.45$ & $16.70 \pm 1.75$ \\
\hline 4 & Ethanol 200 (200 mg/kg p.o) & $75.50 \pm 3.26$ & $15.80 \pm 1.42$ \\
\hline 5 & Ethanol $100(100 \mathrm{mg} / \mathrm{kg}$ p.o $)$ & $25.80 \pm 3.07$ & $13.60 \pm 0.92$ \\
\hline 6 & Water 300 (300 mg/kg p.o) & $59.20 \pm 3.35$ & $14.40 \pm 1.75$ \\
\hline 7 & Water 200 (200 mg/kg p.o) & $50.20 \pm 4.98$ & $16.30 \pm 1.65$ \\
\hline 8 & Water 100 (100 mg/kg p.o $)$ & $25.70 \pm 3.23$ & $13.50 \pm 1.61$ \\
\hline
\end{tabular}
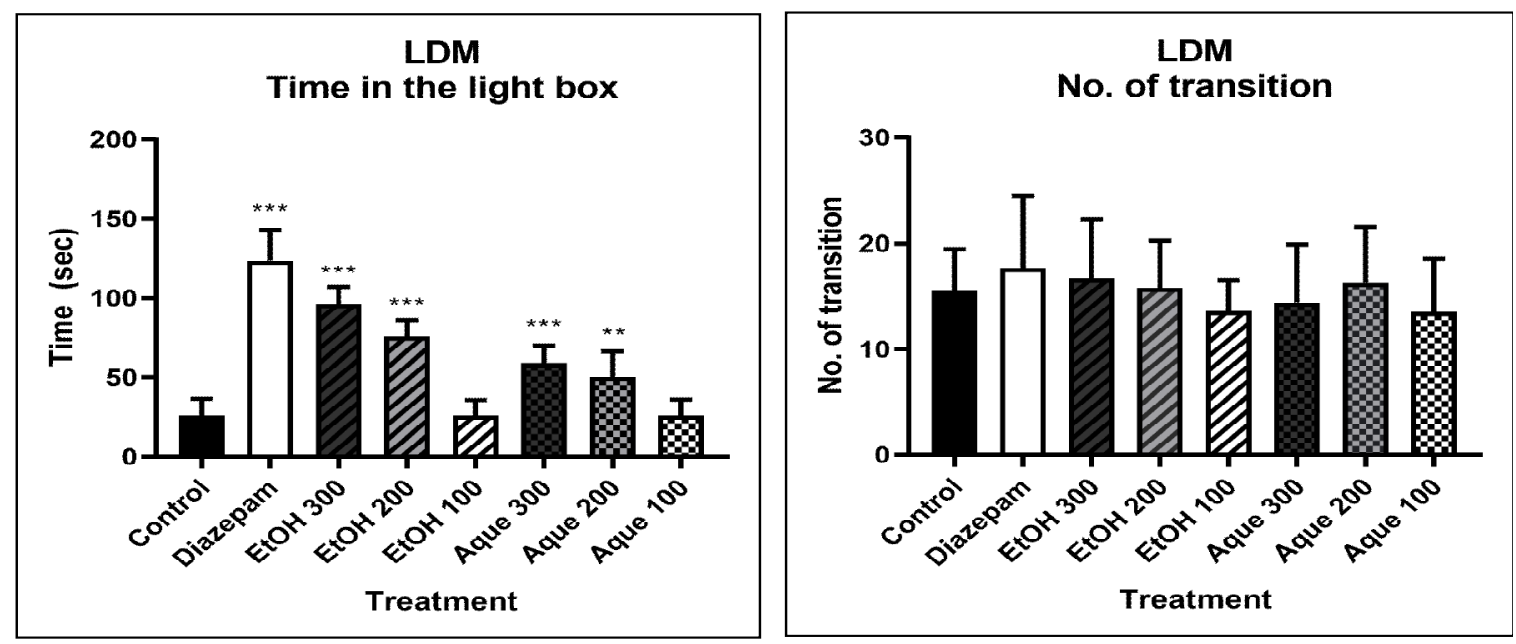

Figure 2. Light dark test

\section{CONCLUSION}

The above research results are the basis for us to make appropriate decisions in the selection of suitable extraction solvents, dosage and pharmaceutical techniques to bring optimal sedative effects for the sedative and anxiolytic tea bags extracted from PE and to conduct further studies on anxiolytic and sedative effect of pharmaceutical products in the future.

\section{REFERENCES}

1. Yadav, A.V., L.A. Kawale, and V.S. Nade, Effect of Morus alba L. (mulberry) leaves on anxiety in mice. Indian J Pharmacol, 2008. 40(1): p. 32-6.

2. Asnis, G.M., M. Thomas, and M.A. Henderson, Pharmacotherapy Treatment Options for Insomnia: A Primer for Clinicians. Int J Mol Sci, 2015. 17(1).

3. Lader, M. and S. Morton, Benzodiazepine problems. Br J Addict, 1991. 86(7): p. 823-8.

4. Liu, L., et al., Herbal Medicine for Anxiety, Depression and Insomnia. Curr Neuropharmacol, 2015. 13(4): p. 48193.

5. Taïwe, G. and V. Kuete, Passiflora edulis, in Medicinal Spices and Vegetables from Africa. 2017, Elsevier. p. 513526.
6. Barbosa Santos, T., et al., Phytochemical Compounds and Antioxidant Activity of the Pulp of Two Brazilian Passion Fruit Species: Passiflora Cincinnata Mast. And Passiflora Edulis Sims. 2021: p. 1-15.

7. Dhawan, K., S. Dhawan, and A.J.J.o.e. Sharma, Passiflora: a review update. 2004. 94(1): p. 1-23.

8. Tiwari, P., et al., Phytochemical screening and extraction: a review. 2011. 1(1): p. 98-106.

9. Lister, R.G., Ethologically-based animal models of anxiety disorders. Pharmacol Ther, 1990. 46(3): p. 321-40.

10. Bourin, M. and M. Hascoët, The mouse light/dark box test. Eur J Pharmacol, 2003. 463(1-3): p. 55-65.

11. He, M., et al., A review on the pharmacological effects of vitexin and isovitexin. 2016. 115: p. 74-85.

12. Patel, S., et al., Passiflora incarnata Linn: A phytopharmacological review. International Journal of Green Pharmacy, 2009. 3.

13. Shinomiya, K., et al., Hypnotic activities of chamomile and passiflora extracts in sleep-disturbed rats. 2005. 28(5): p. 808-810.

14. Park, S.-H., et al., Antidepressant-like Effect of Kaempferol and Quercitirin, Isolated from Opuntia ficus-indica var. saboten. 2010. 19(1): p. 30. 
15. Aguirre-Hernández, E., et al., HPLC/MS analysis and anxiolytic-like effect of quercetin and kaempferol flavonoids from Tilia americana var. mexicana. 2010. 127(1): p. 91-97.

16. Aricioglu, F. and H.J.A.o.t.N.Y.A.o.S. Altunbas, Harmane Induces Anxiolysis and Antidepressant-Like Effects in Rats. 2003. 1009(1): p. 196-201.

17. Lister, R.G.J.P., The use of a plus-maze to measure anxiety in the mouse. 1987. 92(2): p. 180-185.
18. Pellow, S., et al., Validation of open:closed arm entries in an elevated plus-maze as a measure of anxiety in the rat. J Neurosci Methods, 1985. 14(3): p. 149-67.

19. Young, R., D.N.J.P.B. Johnson, and Behavior, A fully automated light/dark apparatus useful for comparing anxiolytic agents. 1991. 40(4): p. 739-743.

20. Reagan-Shaw, S., M. Nihal, and N.J.T.F.j. Ahmad, Dose translation from animal to human studies revisited. 2008. 22(3): p. 659-661.

Citation: Hung Tran - Quoc, Sy Duong-Quy, et al., "Evaluation of Phytochemical Constituents and Sedative Activity of Passiflora edulis Sims Extracts”, American Research Journal of Pharmacy, Vol 6, no. 1, 2021, pp. 1-6.

Copyright (C) 2021 Hung Tran - Quoc, Sy Duong-Quy, et al., This is an open access article distributed under the Creative Commons Attribution License, which permits unrestricted use, distribution, and reproduction in any medium, provided the original work is properly cited. 